\section{Infant growth and childhood respiratory health: how long is the road to preventive measures?}

\author{
Maja Popovic
}

Over the past several decades, longitudinal studies, and especially more recent birth cohorts, have made landmark contributions toward clarifying the association between obesity and asthma. Several possible biological mechanisms have been suggested and the consistency of the association with the temporal and dose-response link have been replicated in a wide variety of settings, including different age groups, cultural, ethnic, socioeconomic and geographic backgrounds. Nowadays, it is largely recognised that obesity is implicated in asthma development, ${ }^{1}$ but the road to the identification of environmental and behavioural modifiable risk factors and quantification of health benefits related to obesity prevention is still steep and long.

As asthma is a complex and heterogeneous disease that usually begins in early childhood, and in line with 'fetal origins' hypothesis, ${ }^{2}$ the current research has moved the interest to exposures during prenatal and early postnatal period, and in particular to fetal and infant weight and growth. It was reasonable to assume that biologically plausible mechanisms proposed for childhood and adulthood obesity ${ }^{1}$ could be extended also to the effect of fetal and infant weight on lung development and its later function. These include inflammatory and immune responses, obesity-related reductions in pulmonary compliance and limitations in airflow, and, more recently, disrupted microbiota. ${ }^{1}$

Almost 30 years ago, Barker et $a l^{3}$ reported low birth weight to be associated with reduced adult lung function, and since then this association has been replicated in children and in adults. Low birth weight, which is the most commonly used proxy of intrauterine growth restriction and a strong predictor of fast postnatal growth, is a well-established risk factor for childhood and adulthood respiratory morbidity. However, some disagreement still exists on how much of this association is explained by gestational age. ${ }^{45}$

Correspondence to Dr Maja Popovic, Department of Medical Sciences, University of Turin, Turin 10126, Italy; mpopovic@unito.it
Interestingly, current evidence supporting the associations of fetal growth with childhood asthma and lung function is less conclusive. ${ }^{6-9}$ This could be, at least in part, due to limitations of methods for measuring intrauterine growth and different growth dimensions measured. Studies that examined postnatal growth suggest that rapid weight growth in the first years of life increases the risk of childhood wheeze, asthma and lung function decline, ${ }^{4} 67$ 9-13 while less consistent associations were found for early height growth. 81011 Application of complex methods to longitudinally model growth in early childhood enabled capturing subtle differences and more complex growth dimensions, suggesting that not only the increased mean weight, but also the rate/ velocity of weight growth and the timing of weight growth peak might contribute to later respiratory morbidity. ${ }^{10} 13$

In Thorax, Casas et $a l^{14}$ report a well-designed and comprehensive analysis of the associations of several weight and height growth parameters in the first 3 years of life with childhood asthma and lung function parameters of lung volume and airway obstruction. Using longitudinally and objectively assessed growth and lung volumes data on 4435 children from the Generation R cohort, they found that children with greater peak weight velocity and higher body mass index (BMI) at adiposity peak in the first years of life had an increase in lung volumes and reduced airway patency at 10 years of age. Early height growth was not associated with childhood lung function in the current study. Consistently with several previous studies, ${ }^{9} 1012$ these findings are characterised by relatively small increase in Z-score of forced vital capacity (FVC) and forced expiratory volume in $1 \mathrm{~s}\left(\mathrm{FEV}_{1}\right)$ ( \pm 0.05 change in $\mathrm{z}$-score), and similar small reductions in $\mathrm{z}$-score of $\mathrm{FEV}_{1} / \mathrm{FVC}$ and forced expiratory flow 75\% $\left(\mathrm{FEF}_{75}\right)$. While there is a clear evidence for the and lung function, one might question its clinical relevance. Although small-magnitude effect sizes could have important implications for later health, it needs to be recognised that the observed relationship association between early weight growth is evidently a weak one, and that factors other than early weight growth per se could be more relevant for childhood lung function.

This study is based on a large birth cohort that has an advantage of having prospectively and objectively measured weight, height and lung function parameters. Extensive growth measures were collected at multiple time points allowing application of complex models to precisely estimate individual growth trajectories. The overall results were robust to adjustment for a number of confounding factors and sensitivity analyses, and several potential effect modifiers were also tested. Surprisingly, the authors observed no association of early weight growth with asthma at 10 years of age. Previous studies were consistent in observing an increased risk of wheeze and asthma in relationship with early weight growth, both in preschoolers and in school-aged children. $\begin{array}{llll}4 & 6 & 10-13\end{array}$ The lack of association with school-age asthma has been reported by one study conducted in the same population of children from the Generation R cohort, while in the same children an association with preschool wheezing and asthma has been reported. ${ }^{715}$ Despite the careful definition that combined self-reported physician-diagnosed asthma with either wheezing or inhalant medication use in the past 12 months, the outcome misclassification cannot be completely ruled out. Therefore, the authors' claim of potentially different mechanism involved in the association with lung function compared with the one with asthma is plausible but not convincing. Also, the sex-stratified analyses showed that the associations of age at adiposity peak with asthma and lung function were present among boys but not among girls. Sex differences have been often reported in studies of growth, obesity and asthma, but with conflicting results and many open questions.

In addition to largely confirmatory findings on the link between early weight growth and lung function, and somewhat contrasting results on asthma, Casas et $a l^{14}$ went beyond common association analyses and opened a welcome new 'black box' of the potential mediators in the early growth-respiratory morbidity pathway. Recent advancements in the causal inference methods enhanced our curiosity about the mechanisms by which effects are transmitted, especially in situations when mechanistic studies are unethical but experimental manipulations targeting the mediator are possible. Mediation analysis methods assess the relative magnitude of different pathways/mechanisms by which 
an exposure may affect an outcome. ${ }^{16}$ These methods consist of statistical techniques aimed at decomposing the total effect (the observed relationship between an exposure and an outcome) into a direct (unmediated) effect and an indirect effect mediated by a mediator. ${ }^{16-18}$ The current study ${ }^{14}$ explored the two plausible indirect pathways, including preschool wheezing disorders and childhood current BMI status. While preschool wheezing did not meet the predefined criteria for mediation analysis used in this study, the authors found that the positive association between early weight growth parameters and BMI at 10 years of age largely explains the associations of early weight growth with increased lung volumes and a small part of the associations with reduced airway patency.

This interesting finding brings us back to the obesity prevention, modifiable factors and strategies for improving obesity-related health outcomes. If childhood BMI really lays on the pathway between early growth and lung function, then many preventive measures have already been identified. Still, this is only a first piece of the complex puzzle that needs to be solved in the future. Before moving forward, it is essential to understand that mediation analysis is not a solution but just an approach that needs to be used with all its advantages and limitations. Establishing mediating pathways by which an effect is transmitted is challenging, and strong requirements are needed in terms of the temporal ordering, unmeasured confounders and model specification. ${ }^{16}$ It becomes clear that the way to the strategies for reducing the burden of fast infant growth, in which we have already made important steps, will be a long road trip with many turns and intersections.

We now know that prenatal and early life are the most critical period for interventions and that postnatal weight growth likely plays a role in childhood lung function and asthma onset. We also know that the effects of growth on respiratory health are evident already from preschool period and persist until adolescence. What is still unclear are the mechanisms by which rapid growth and large body size affect lung development and function; there is also a debate whether the changes observed until adolescence are reversible or persist in adulthood, and we still do not know which interventions and to what extent our actions could improve later respiratory health. The study by Casas et $a l^{14}$ is a reminder that many questions are still to be answered, but scientific and technological development, such as advances in the field of molecular epidemiology, sequencing technologies and gut microbiome research, with recent developments in mediation analysis, and better communication between basic and applied science when designing future studies will eventually accelerate the pace we move towards healthier lungs.

Funding The authors have not declared a specific grant for this research from any funding agency in the public, commercial or not-for-profit sectors.

Competing interests None declared.

Patient consent Not required.

Provenance and peer review Commissioned; externally peer reviewed.

(c) Author(s) (or their employer(s)) 2018. No commercial re-use. See rights and permissions. Published by BMJ.

\section{Check for updates}

To cite Popovic M. Thorax 2018;73:1101-1102.

Accepted 10 September 2018

Published Online First 28 September 2018

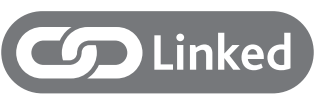

- http://dx.doi.org/10.1136/thoraxjnl-2017-211216

Thorax 2018;73:1101-1102.

doi:10.1136/thoraxjnl-2018-212341

\section{REFERENCES}

1 Peters U, Dixon AE, Forno E. Obesity and asthma. J Allergy Clin Immunol 2018;141:1169-79.

2 Barker DJ. Fetal origins of coronary heart disease. BMJ 1995;311:171-4.
3 Barker DJ, Godfrey KM, Fall C, et al. Relation of birth weight and childhood respiratory infection to adult lung function and death from chronic obstructive airways disease. BMJ 1991:303:671-5.

4 Sonnenschein-van der Voort AM, Arends LR, de Jongste JC, et al. Preterm birth, infant weight gain, and childhood asthma risk: a meta-analysis of 147,000 European children. J Allergy Clin Immunol 2014;133:1317-29.

5 Balte $\mathrm{P}$, Karmaus W, Roberts G, et al. Relationship between birth weight, maternal smoking during pregnancy and childhood and adolescent lung function: A path analysis. Respir Med 2016;121:13-20.

6 Pike KC, Crozier SR, Lucas JS, et al. Patterns of fetal and infant growth are related to atopy and wheezing disorders at age 3 years. Thorax 2010;65:1099-106.

7 Sonnenschein-van der Voort AM, Jaddoe VW, Raat H, et al. Fetal and infant growth and asthma symptoms in preschool children: the generation R study. Am J Respir Crit Care Med 2012;185:731-7.

8 Turner S, Fielding S, Devereux G. First trimester fetal size and prescribed asthma medication at 15 years of age. Eur Respir J 2018;51:1701509.

9 den Dekker HT, Jaddoe VWV, Reiss IK, et al. Fetal and infant growth patterns and risk of lower lung function and asthma. the generation R study. Am J Respir Crit Care Med 2018:197:183-92.

10 Sonnenschein-van der Voort AM, Howe LD, Granell R, et al. Influence of childhood growth on asthma and lung function in adolescence. J Allergy Clin Immunol 2015;135:1435-43.

11 Magnus MC, Stigum H, Håberg SE, et al. Peak weight and height velocity to age 36 months and asthma development: the Norwegian Mother and Child Cohort Study. PLoS One 2015;10:e0116362.

12 den Dekker HT, Sonnenschein-van der Voort AMM, de Jongste JC, et al. Early growth characteristics and the risk of reduced lung function and asthma: a metaanalysis of 25,000 children. J Allergy Clin Immunol 2016:137:1026-35.

13 Popovic M, Pizzi C, Rusconi F, et al. Infant weight trajectories and early childhood wheezing: the NINFEA birth cohort study. Thorax 2016;71:1091-6.

14 Casas M, den Dekker HT, Kruithof CJ, et al. The effect of early growth patterns and lung function on the development of childhood asthma: a population based study. Thorax: [Published Online First: 31 Jul 2018 doi: 10.1136/thoraxjnl-2017-211216].

15 Casas M, den Dekker HT, Kruithof CJ, et al. Early childhood growth patterns and school-age respiratory resistance, fractional exhaled nitric oxide and asthma. Pediatr Allergy Immunol 2016;27:854-60.

16 VanderWeele TJ. Explanation in causal inference: Methods for mediation and interaction. New York, NY: Oxford University Press, 2015.

17 Zugna D, Richiardi L. [Effects decomposition in mediation analysis: a numerical example]. Epidemiol Prev 2018;42:127-33.

18 Richiardi L, Bellocco R, Zugna D. Mediation analysis in epidemiology: methods, interpretation and bias. Int J Epidemiol 2013;42:1511-9. 